\title{
Inhibition of JNK increases survival of transplanted dopamine neurons in Parkinsonian rats
}

\author{
Cell Death and Differentiation (2007) 14, 381-383. doi:10.1038/sj.cdd.4402010; published online 21 July 2006
}

Dear Editor,

Parkinson's disease (PD) affects approximately $2 \%$ of the population over 65 years of age. ${ }^{1}$ This neurodegenerative disorder is caused by a selective and progressive death of the dopaminergic (DA) neurons of the substantia nigra and subsequent striatal deficiency of dopamine, resulting in movement anomalies. Vast efforts are being made to develop cell replacement therapies using fetal transplantation and stem cell based approaches to improve treatment. Proof of concept for transplantation of human fetal mesencephalic tissue into the striatum of PD patients was obtained in $1987 .^{2-5}$ However, several issues have limited the use of fetal transplants in a clinical setting, including the appearance of dyskinesias, ethical considerations, tissue preparation and storage, distribution and site of graft, immunosupression, and limited availability of fetal tissue. ${ }^{6-8}$ Approximately 250000 DA cells reside in the adult human substantia nigra but only 20000-40000 DA neurons can be obtained from a single fetus, making it necessary to obtain tissue from up to $6-8$ fetuses per patient. ${ }^{5}$ Reports have shown that only $3-5 \%$ of fetal DA neurons survive upon transplantation into rodent models of PD. ${ }^{9}$ Moreover, a similar problem has been described for the transplantation of human embryonic stem cell derived DA neurons. ${ }^{10}$ Thus, improving the survival of DA neurons during isolation as well as post-transplantation has become a central issue. Cell death during grafting can be observed throughout several phases: dissection and preparation of tissue, the implantation procedure, and immediately or long-term after graft injection. However, the largest death associated with transplantation of fetal tissue in rodent models of PD occurs during the first week post-transplantation. ${ }^{11,12}$ We therefore decided to examine the possibility of reducing DA cell death during this period. Caspase inhibitors have been reported to increase the survival of transplanted tissue and thereby result in functional improvement. ${ }^{13}$ Importantly, however, these compounds lack specificity for DA neurons, thereby increasing the total survival of the graft, including nonDA cells. In this regard, we examined the function of C-Jun-Nterminal kinase (JNK) and its role in early DA cell death. JNK is activated in response to cellular stress and DNA damage and can activate caspases, resulting in apoptosis. Activated JNK has been shown in neuronal cell death both in vitro and in vivo, ${ }^{14}$ and increased levels of phospho c-Jun, a downstream mediator of JNK signaling, have been reported in rodent models of PD. ${ }^{15-18}$ In order to assess whether inhibition of JNK could modulate the numbers of DA neurons, we treated ventral midbrain (VM) embryonic day (E) 13.5 and E14.5 precursor cultures with increasing doses of the JNK inhibitor SP600125 $5^{19}$ for 1 or 3 days in vitro (1 DIV or 3 DIV). Addition of SP600125 to the precursor cultures increased the number of DA neurons $(\mathrm{TH}+)$ in a dose-dependent manner, with maximal effects at $15 \mu \mathrm{M}$. In the $\mathrm{E} 13.5$ cultures, $15 \mu \mathrm{M}$ SP600125 increased the number of $\mathrm{TH}+$ neurons per field by nine-fold at 1DIV and by 13-fold at 3DIV. In the E14.5 cultures, when more DA neurons have been born, $15 \mu \mathrm{M}$ SP600125 increased the number of $\mathrm{TH}+$ neurons per field by two-fold at 1DIV and by eight-fold at 3DIV (Figure 1a(i)). The biggest increase (13-fold) was observed in E13.5 cells cultured for 3DIV (Figure $1 \mathrm{a}(\mathrm{ii})$ ) and the highest yield of $\mathrm{TH}+$ neurons was obtained from E14.5 tissue cultured for 1DIV (approximately 7.5 TH + cells/field). Upon culturing for 7DIV, the neurons develop into mature DA neurons, as assessed by immunoreactivity against the dopamine transporter protein (data not shown). The observation that the largest effect was observed in the E13.5 cultures, when few DA neurons have been generated, suggests that the increase in DA numbers might also be ascribed to effects on progenitors restricted to the DA lineage. The effect of SP600125 on E13.5 3DIV cultures was confirmed using a cell permeable JNK inhibitor peptide, which also resulted in a significant increase in the number of $\mathrm{TH}+$ neurons, compared to a control peptide ( $P$-value $<0.004)(3 \mu \mathrm{M}$ respectively, Calbiochem No. 420116, 420118). In order to address the specificity of SP600125, we analyzed the effects on the predominant cell populations present in the culture. We found that the total number of cells, as assessed by Hoechst 33258, was not altered (control: $195.6 \pm 10.24$, SP600125 197.1 \pm 16.71 ), nor was the neuronal fraction, as assessed by $\beta$ III-tubulin/Hoechst 33258 (control: $0.40 \pm 0.05$, SP600125 $0.57 \pm 0.11$ ). Interestingly, however, the number of DA neurons out of the total number of neurons, as assessed by TH/Tuj1, increased by 11-fold (Figures $1 b(i)$ and $b(i i)$ ). Inhibition of the JNK pathway has been described to have anti-apoptotic effects on neuronal cells. ${ }^{14}$ We thus examined the effects of JNK inhibition in our precursor cultures and found that the number of active Caspase 3 immunoreactive cells was significantly reduced upon addition of $15 \mu \mathrm{M}$ SP600125 (control: 23.46 \pm 1.00 , SP600125: $12.10 \pm 1.69$ ) (Figure 1c). In support of our findings, administration of SP600125 has been shown to protect DA neurons in an MPTP model of PD. ${ }^{18}$ In order to further address the effects of JNK inhibition on downstream signaling components, we treated E13.5 cultures with $15 \mu \mathrm{M}$ SP600125 and observed a statistically significant reduction in the levels of phospho-c-Jun, a marker of activated apoptotic JNK signaling (Figure 1d). Combined, these results suggest that apoptotic JNK signaling is more relevant during the generation and maintenance of DA neurons than in the total neuronal pool. Since JNK inhibitors have been found to protect adult DA neurons in an MPTP model of PD, ${ }^{17,18}$ 
ai

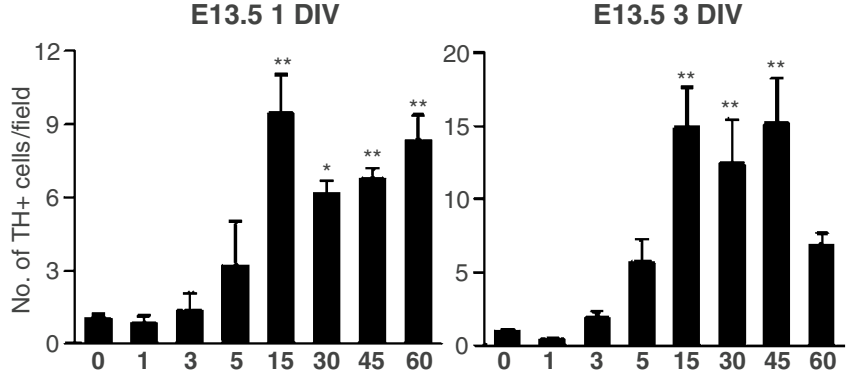

E14.5 1 DIV

E14.5 3 DIV

aii

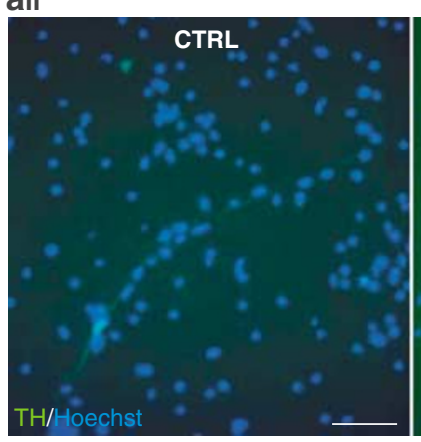

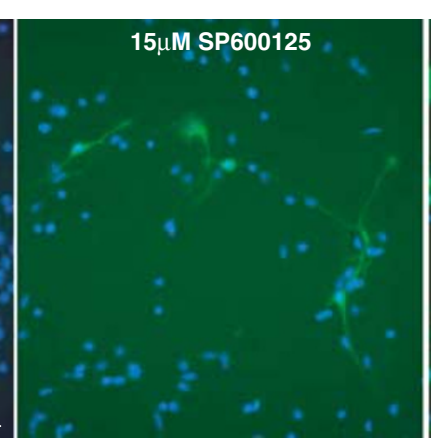

bi

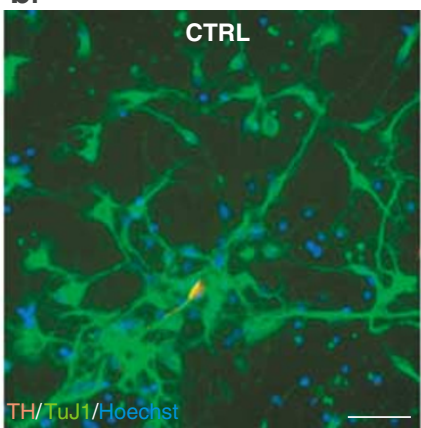

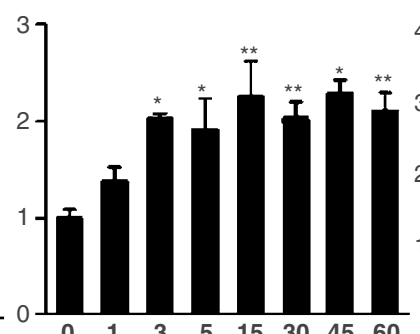
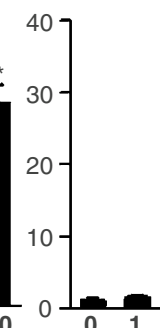

bii

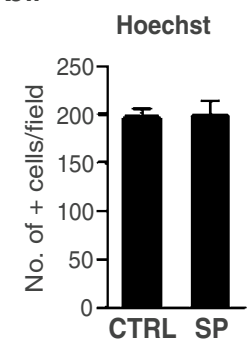

ei

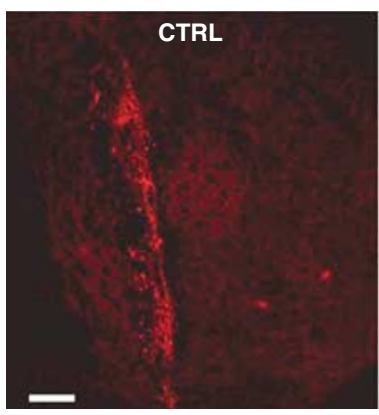

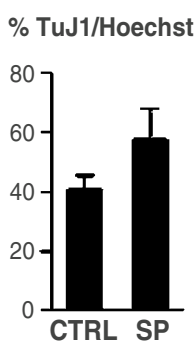

$\%$ TH/TuJ1

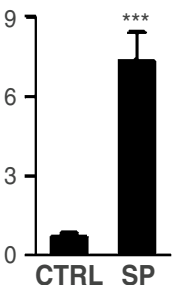

Caspase3

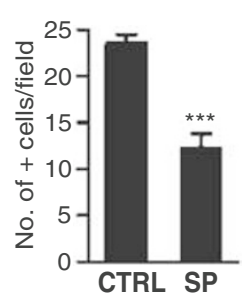

eii

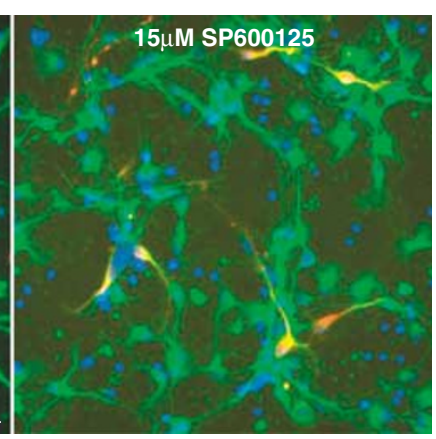

d

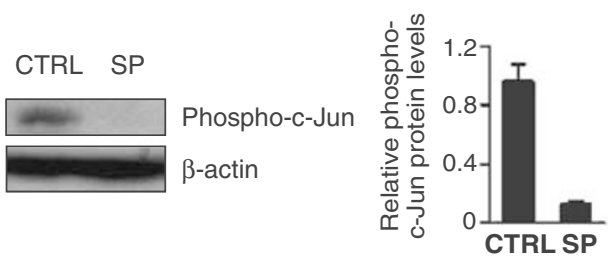

f

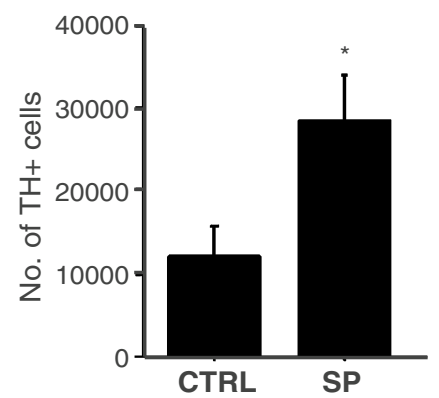

Figure 1 SP600125 specifically increases the number of tyrosine hydroxylase positive $(\mathrm{TH}+)$ neurons in VM and increases graft survival and TH + cell numbers following transplantation of primary VM cells into Parkinsonian rats. (a(i)) Dose-response experiments in E13.5 and E14.5 VM precursors, at 1 day in vitro (DIV) and 3DIV indicate that $15 \mu \mathrm{M}$ SP600125 (SP) is the optimal dose to increase TH + cell numbers. The biggest increase in the number of TH + cells was observed in the E13.5 3 DIV condition. Statistical analysis was performed using a one-way ANOVA with Dunnett's multiple comparison test. (a(ii)) TH/Hoechst 33258 immunostaining of an E13.5 progenitor culture shows an increase in the number of TH immunoreactive cells 3 days after treatment with $15 \mu \mathrm{M} \mathrm{SP600125.} \mathrm{Bar:} 50 \mu \mathrm{m}$. (b(i), $\mathbf{b}$ (ii)) Treatment with SP does not alter the total number of cells present in the culture, as assessed by Hoechst 33258 , nor the total neural fraction, as assessed by Tuj1/Hoechst 33258 . However, the inhibitor significantly increases the DA fraction out of the total neuronal population, as assessed by TH/Tuj1, while it significantly reduces the number of Caspase 3 immunoreactive cells (c). Statistical analysis was performed using a two-tailed unpaired Student's $t$-test. Bar: $50 \mu \mathrm{m}$. (d) Treatment of E13.5 cultures with $15 \mu \mathrm{M} \mathrm{SP}$ for $24 \mathrm{~h}$ results in decreased JNK signaling, as assessed by protein levels of phospho-c-Jun. $\beta$-Actin was used as a protein loading control and the graph depicts relative phospho-c-Jun levels, normalized against $\beta$-actin protein levels. (e(i)) Photomicrograph of vehicle and SP600125 treated grafts respectively. Bar: $500 \mu \mathrm{m}$ (e(ii)) High magnification picture of grafted cells immunoreactive for TH, illustrating a typical bipolar morphology. Bar: $50 \mu$ m. (f) Intraventricular infusion of $75 \mu \mathrm{M}$ SP600125 post-transplantation of fetal VM primary cells results in an increased number of striatal TH + cells compared to infusion of DMSO vehicle. Data in (f) represent the mean \pm S.E.M. Statistical analysis was performed using a two-tailed unpaired Student's $t$-test 
we next examined the effect of SP600125 on grafted embryonic DA neurons in vivo, in an animal model of PD. We unilaterally injected the neurotoxin 6-OHDA into the right SNpc of rats to produce a complete ablation of cell bodies as well as fibers and terminals innervating the striatum. Based on previous findings, ${ }^{20}$ all animals showing greater than 5 rotations per minute were assumed to have lesions greater than $90 \%$ and were selected for transplantation. At 2 weeks post-lesioning, $400000 \mathrm{VM}$ cells were treated with vehicle or SP600125 and grafted into the striatum of lesioned rats. ${ }^{20}$ Animals received daily infusions of vehicle or SP600125 $(75 \mu \mathrm{M})$ via a cannula in the striatum for 4 days and were killed 7 days post-transplantation. Striatal examination revealed that $100 \%$ of the VM grafts survived in animals infused with either vehicle or SP600125. However, total counts of $\mathrm{TH}+$ cells in the grafts of animals infused with SP600125 showed significantly more (231\%) DA cells than in the grafts of animals infused with vehicle (mean \pm S.E.M, $12285 \pm 3436$ and $28406 \pm 5506$, respectively, Figures $1 \mathrm{e}(\mathrm{i})$ and (f)). Thus, treatment of cells with SP600125 upon transplantation into 6-OHDA lesioned brains results in a two-fold increase in the survival of grafted DA neurons within the host striatum. Moreover, we found that within 7 days, these cells had adopted a typical bipolar DA phenotype with extensive processes, verifying that the cells incorporated within the host and did not form tumors (Figure 1e(ii)). These results indicate that SP600125 selectively prevents cell death, and does not induce excessive proliferation in vivo. From a clinical viewpoint, our results imply that the amount of DA precursors needed for transplantation in PD could be at least halved by using the SP600125 compound. Taken together, our study indicates that inhibition of apoptotic JNK signaling may contribute to overcome one of the major limitations of current cell replacement strategies in the treatment of PD.
Acknowledgements. We thank Dr. Tibor Harkany for fruitful discussions, Dr. Björn Schilström for technical advice and Claudia Tellos-Lagos for technical assistance. Financial support was obtained from the Swedish Foundation for Strategic Research, EuroStemCell, Swedish Royal Academy of Sciences, Knut and Alice Wallenberg Foundation, and the Karolinska Institute. CP was supported by the Human Frontiers Scientific Program Longterm Fellowship, and GCB was supported by the Praxis XXI programme of the Portuguese Fundação para a Ciência e Tecnologia/European Social Fund, the Karolinska Institute, and Calouste Gulbenkian Foundation.

\section{N Rawal ${ }^{1}, C$ Parish $^{1,2}$, G Castelo-Branco ${ }^{1,2}$ and E Arenas $^{* 1}$}

${ }^{1}$ Laboratory of Molecular Neurobiology, Department of Medical Biochemistry and Biophysics, Karolinska Institute, Scheeles v. 1, A1:2, 17177 Stockholm Sweden

2 These authors contributed equally to this work.

* Corresponding author: E Arenas, Laboratory of Molecular Neurobiology, Department of Medical Biochemistry and Biophysics, Karolinska Institute, Scheeles v. 1, A1:2, 17177 Stockholm, Sweden. Tel: + 46852487663; Fax: + 468341960; E-mail: Ernest.Arenas@ki.se

1. de Rijk MC et al. Neurology 2000; 54 (Suppl 5): S21-S23.

2. Lindvall $O$ et al. Ann Neurol 1994; 35: 172-180.

3. Piccini $P$ et al. Nat Neurosci 1999; 2: 1137-1140.

4. Wenning GK et al. Ann Neurol 1997; 42: 95-107.

5. Olanow CW et al. Trends Neurosci 1996; 19: 102-109.

6. Lindvall O, Bjorklund A. NeuroRx 2004; 1: 382-393.

7. Madrazo I et al. N Engl J Med 1988; 318: 51

8. Quinn NP. Prog Brain Res 1990; 82: 619-625

9. Brundin $P$ et al. Cell Transplant 2000; 9: 179-195

10. Zeng X et al. Stem Cells 2004; 22: 925-940.

11. Barker RA et al. Exp Neurol 1996; 141: 79-93.

12. Zawada WM et al. Brain Res 1998; 786: 96-103.

13. Schierle GS et al. Nat Med 1999; 5: 97-100.

14. Silva RM et al. Mov Disord 2005; 20: 653-664.

15. Holtz WA, O'Malley KL. J Biol Chem 2003; 278: 19367-19377.

16. Vaudano E, Rosenblad C, Bjorklund A. Eur J Neurosci 2001; 13: 1-14.

17. Xia XG et al. Proc Natl Acad Sci USA 2001; 98: 10433-10438.

18. Wang W et al. Neurosci Res 2004; 48: 195-202.

19. Bennett BL et al. Proc Natl Acad Sci USA 2001; 98: 13681-13686.

20. Parish CL et al. Stem Cells 2005; 23: 471-476.

\title{
Mitochondrial membrane depolarization in Drosophila apoptosis
}

\author{
Cell Death and Differentiation (2007) 14, 383-385. doi:10.1038/sj.cdd.4402036; published online 15 September 2006
}

\section{Dear Editor}

Apoptosis regulation in Drosophila has many parallels to vertebrate systems. However, one major difference has been the apparent lack of mitochondrial significance in Drosophila cell death. Several reports have suggested that mitochondrial outer membrane permeabilization does not occur in Drosophila apoptosis and that there is no significant mitochondrial contribution to apoptosis in the fly. ${ }^{1-6}$ However, these findings are based largely, if not exclusively, on studies in cultured S2 cells, which are phagocytic cells derived from embryonic macrophages/hemocytes. ${ }^{7,8}$ The specialized nature of S2 cells prompted us to consider whether they are generally representative of Drosophila cells in their response to apoptotic stimuli. We addressed this question by examining the response of embryonic macrophages to apoptotic signals in situ in the embryo.

In keeping with previous accounts, we find that apoptotic S2 cells maintain mitochondrial membrane integrity as measured by CMXRos staining. ${ }^{4}$ CMXRos is a mitochondrial-specific label dependent on membrane polarization for uptake. Untreated cells show punctate cytoplasmic staining consistent 